\title{
Morbidity patterns in general practice settings of the province of Sousse, Tunisia
}

\author{
Ridha Gataa ${ }^{1 \&}$, Thouraya Nabli Ajmi ${ }^{1}$, I heb Bougmiza ${ }^{1}$, Ali Mtiraoui ${ }^{1}$
}

${ }^{1}$ Department of Community Medicine - Medical Faculty of Sousse (Tunisia)

${ }^{\&}$ Corresponding author: Faculté de Médicine - Avenue Mohamed Karoui - Sousse 4000 - Tunisie. Tel: +216 - 98503933 or +216 - 73220675 , Fax: $+216-73210925$.

Key words: General practice, problems managed by general practitioners, International Classification of Primary Care, ICPC-2

Received: 03/11/2008 - Accepted: 20/10/2009 - Published: 02/11/2009

Pan African Medical J ournal, 2009 3:11

This article is available online at: http://www.panafrican-med-journal.com/content/article/3/11/full

(c) Ridha Gataa et al. The Pan African Medical J ournal - ISSN 1937-8688. This is an Open Access article distributed under the terms of the Creative Commons Attribution License (http://creativecommons.org/licenses/by/2.0), which permits unrestricted use, distribution, and reproduction in any medium, provided the original work is properly cited.

\begin{abstract}
Background: Primary health care is one of the most important pillars of the Tunisian health care system. However, very little information is available regarding the specificities of general practice and the patterns of morbidity encountered. Methods: We conducted a descriptive study from June 2002 to May 2003 in 85 primary health centres in Sousse during 12 randomly selected weeks in order to describe the variability of the morbidity in all seasons; ( 3 weeks were randomly selected in each season). Each working day of selected weeks, a systematic sample of patients was identified in each health centre by taking every fifth registered patient. There were 16,271 consultations. The International Classification of Primary Care (ICPC-2) was used to code recorded data of the consultation. Results: There were 24,882 reasons for encounter, a total of 18,097 problems managed by general practitioners (GPs), and 40,190 interventions. There was a predominance of females (62\%) and a relatively young population attending the primary health care settings as $50 \%$ was aged less than 25 years. According to ICPC-2 chapters, we found that respiratory diseases were the main problems managed in primary health care $(43 \%)$, followed by digestive $(10.1 \%)$, locomotive $(8.9 \%)$, cardiovascular affections $(8.7 \%)$ and skin diseases $(8.4 \%)$. These five conditions alone constituted about $80 \%$ of the total cases. However, genital conditions for both males and females $(1 \%)$ as well as psychological and social problems $(0.85 \%)$ were rarely managed in primary care. Conclusion: The findings will be useful in helping to revise the educational curriculum of medical studies as required in general practice and to plan relevant vocational training for GPs. They will also be important for health policy makers in Tunisia.
\end{abstract}




\section{Background}

The primary health care system in Tunisia consists of a public sector and a private sector, and both are almost equivalent as regards the number of physicians and consultants. Patients are free to choose the private physician or the public health centre they prefer. Primary health centres are distributed in the whole territory of the country and constitute an important national ambulatory service network. However, very little information is available on the specificities of general practice and the patterns of morbidity encountered. Because another study is underway to learn more about morbidity in private settings, we decided to first investigate the morbidity patterns in the public sector.

The Department of Community Medicine at the Medical Faculty of Sousse carried out, for the first time in Tunisia, this one-year survey in all localities of the province of Sousse, which counted 505,370 inhabitants in 2002. This survey aimed to collect valid, complete, and representative data on reasons for encounter, problems managed by the general practitioner (GP) at the consultation, and interventions in managing these problems in primary health care. However, in this article, we are only considering one aspect of this survey, namely the problems managed by the GP at the consultation in general practice.

Therefore, we considered that the use of the International Classification of Primary Care (ICPC-2) was the most relevant tool for this study. The ICPC-2 allows classification of patient's reasons for encounter (RFE), problems managed as labelled by the GP, and the GPSR17; interventions in managing these problems [1].

The objectives of the study were to illustrate the patterns of morbidity in general practice and to identify the main problems managed by the GPs at the consultation. This study presents no ethical issues; it was conducted in the context of a research unit and with the agreement of the regional health authorities of the Ministry of Health.

\section{Methods}

\section{The study design}

The study design was descriptive and cross-sectional. It was carried out in 85 primary health centres of the province of Sousse (Tunisia) among the 92 existing centres during one year, from J une 2002 to May 2003, covering the four seasons.

\section{Sampling and study population}

The study was conducted during 12 randomly selected weeks in order to describe the variability of the morbidity in all seasons; ( 3 weeks were randomly selected in each season). Each working day of selected weeks, a systematic sample of patients was identified in each health centre by taking every fifth registered patient. There were 16271 consultations, which were described according to a Subjective, Objective, Assessment, Plan (SOAP).

\section{Research instrument}

The data collection instrument was designed to ease the recording process and ensure data quality. A first part was designed to be completed by the nurse in order to record administrative information about the health centre, as well as information describing patient's socio-demographic profile (e.g., sex, age, health insurance). The second part of the instrument was used to collect information resulting from the doctor-patient encounter: reason of encounter, problems managed if any, and any other interventions related to the process of care. The second part was recorded by GPs themselves. All GPs who are working in the health centres were previously trained to conduct this research.

\section{Coding, data entry and analysis}

The International Classification of Primary Care (ICPC-2) was used to code the data according to reasons for encounter, problems managed by the GP, and care related interventions at each consultation. Coding and data computer entry were performed by trained physicians in the department 
of community medicine. We used Epi-info (EPI 6) for data analysis and the significance level considered to determine whether or not the null hypothesis is rejected was $5 \%$.

\section{Results}

A total of 85 out of 92 primary health care centres in the region of Sousse were included as settings for data collection (in 7 health centres, physicians refused to take part of this survey for personal reasons).

\section{Characteristics of the study population}

The population requiring primary health care in the public sector was predominantly female $(62 \%)$. This proportion is significantly higher than in the general population $(P<0.05)$, where the ratio female to male is 1.02 . We found that $74 \%$ of patients were living in urban areas, a similar proportion to that of the general population $(80 \%)(P=0.31)$. The study population was relatively young with a mean age of $31.1 \pm 24.3$ years (the mean age in general population is 28.2 years). Children aged less than 15 years accounted for $33.1 \%$ (of whom $13.3 \%$ were aged less than 1 year), and those aged 65 years and over accounted for $13.7 \%$ of the surveyed consultations (Figure 1 ).

The age-sex distribution of the study population showed that among young people aged less than 25 years, patients were predominantly male. In contrast, patients aged 25-64 years were predominantly female and the sex distribution of those aged 75 years and over was equally distributed (Figure 1).

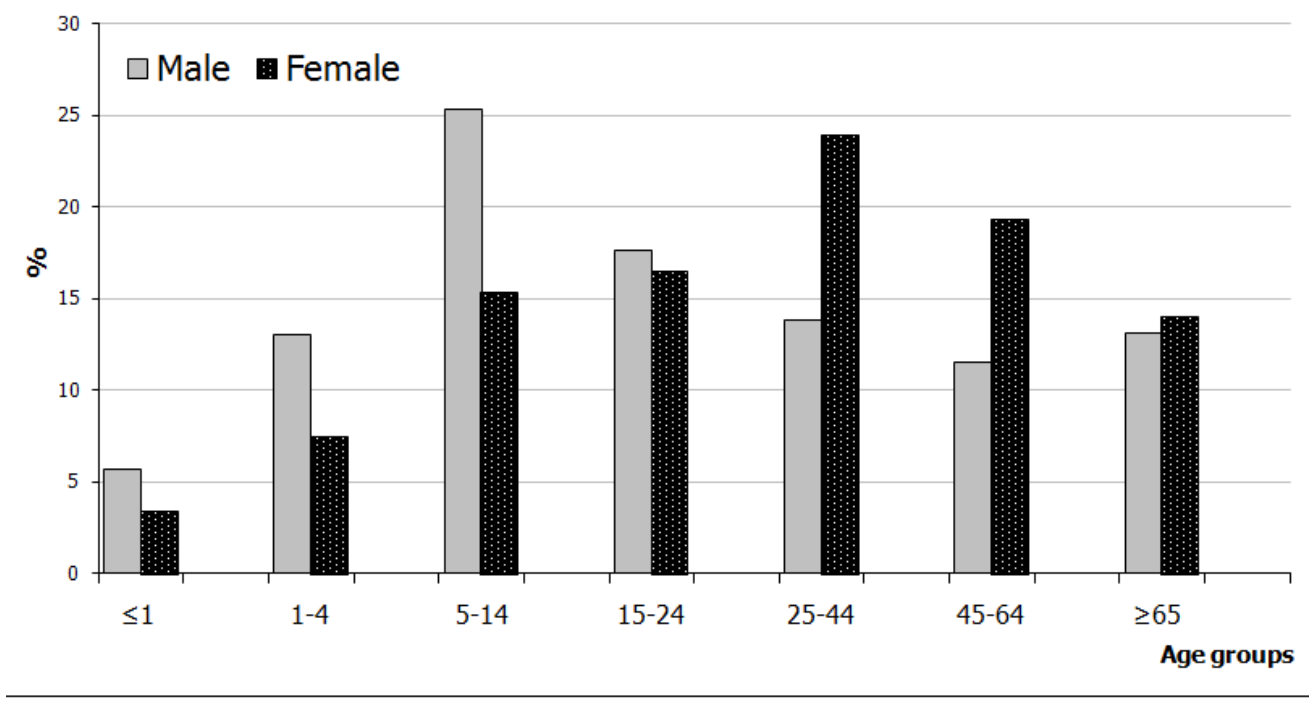

Figure 1: Age and sex distribution of study population

\section{Specificities of primary health care practice}

\section{Characteristics of consultations in general practice}

A total of $81 \%$ of the problems managed were for a new health condition (first episode of care), $13.5 \%$ were for control of a chronic health condition such as hypertension and diabetes, and only $5.5 \%$ of patients were recalled for monitoring an acute illness managed by the GP at a previous consultation. The GPs classified $18 \%$ of the consultations as emergency cases. 


\section{Reasons for encounter, problems managed, and procedures (interventions) in general practice}

There were 24,882 reasons for encounter (RFE) ( $1.9 \pm 0.8$ per encounter), with 18,097 health issues managed as diagnoses ( $1.3 \pm 0.5$ per encounter) and 40,190 interventions (procedures) (3.2 \pm 1.9 per encounter). One RFE was given by the patient in $55.4 \%$ of cases; one single problem was managed in $82.6 \%$ of consultations, while at least two procedures or interventions were undertaken at $67.4 \%$ of consultations.

In $63 \%$ of cases, the GPs decided that no monitoring or control visit was needed for the current episode of care. However, in $31 \%$ of cases, patients were asked to come back for a health control visit and only $5.5 \%$ were referred to a specialist.

\section{Duration of encounters}

The average consultation time was 6.8 minutes $( \pm 3.8)$, and the mode $=$ median $=5$ minutes.

\section{Patterns of health problems managed in primary care}

\section{Health problems classified by I CPC Chapters}

When health problems as managed by the GPs are listed in the order of frequency of the organ systems according to ICPC chapters, we found that respiratory diseases were the main problem managed in general practice $(43 \%)$ followed by digestive $(10.1 \%)$, locomotive (8.9\%), cardiovascular affections $(8.7 \%)$, and skin diseases $(8.4 \%)$. These five affections alone constituted almost $80 \%$ of the total. However, genital affections for both males and females ( $1 \%)$, as well as psychological and social issues $(0.85 \%)$, were rarely identified in primary care (Table 1$)$.

Table 1: Diagnosed morbidity by ICPC Chapter

\begin{tabular}{llcc}
\hline & ICPC Chapters & Frequency & \% \\
\hline R & Respiratory & $\mathbf{7 7 8 8}$ & $\mathbf{4 3 . 0}$ \\
$\mathbf{D}$ & Digestive & 1833 & 10.1 \\
$\mathbf{L}$ & Locomotive (musculoskeletal) & 1611 & 8.9 \\
$\mathbf{K}$ & Cardiovascular & 1574 & 8.7 \\
$\mathbf{S}$ & Skin & 1515 & 8.4 \\
$\mathbf{T}$ & Endocrine and Metabolic & 746 & 4.1 \\
$\mathbf{F}$ & Eye & 631 & 3.5 \\
$\mathbf{A}$ & General & 547 & 3.0 \\
$\mathbf{U}$ & Urological & 479 & 2.6 \\
$\mathbf{H}$ & Ear & 367 & 2.0 \\
$\mathbf{B}$ & Blood & 356 & 2.0 \\
$\mathbf{N}$ & Neurological & 312 & 1.7 \\
$\mathbf{X}$ & Female genital & 141 & 0.8 \\
$\mathbf{P}$ & Psychological & 136 & 0.8 \\
$\mathbf{W}$ & Pregnancy, child-bearing and FP & 31 & 0.2 \\
$\mathbf{Y}$ & Male genital & 22 & 0.1 \\
$\mathbf{Z}$ & Social problems & 8 & 0.0 \\
\hline & Total & $\mathbf{1 8 0 9 7}$ & $\mathbf{1 0 0 . 0}$ \\
\hline
\end{tabular}

\section{Health problems classified by ICPC rubrics}

In addition to acute illnesses (tonsillitis acute: 15.7\%, bronchitis acute: $11.2 \%$, upper respiratory infections: $9.2 \%$, and influenza: 3.5\%), the two most frequent chronic diseases (high blood pressure and diabetes mellitus) were found within the top six, respectively in a proportion of $6.2 \%$ and $3.3 \%$. However, asthma, which seemed to be a common chronic affection in Tunisia, was only managed in a proportion of $0.6 \%$ in general 
practice (Table 2). The non-defined rubrics, as have been classified "others" by ICPC, represented $10.2 \%$ of all health problems managed in primary care.

Table 2: The Top 20 diagnoses according to ICPC Rubrics

\begin{tabular}{|c|c|c|c|c|}
\hline \multirow[t]{2}{*}{ I CPC Code } & \multirow[t]{2}{*}{ I CPC Rubric name } & \multicolumn{3}{|c|}{ Diagnosed Morbidity } \\
\hline & & Frequency & $\%$ & Rank \\
\hline R76 & Tonsillitis acute & 2844 & 15.7 & 1 \\
\hline R78 & Acute bronchitis/bronchiolites & 2032 & 11.2 & 2 \\
\hline R74 & Upper respiratory infection acute & 1659 & 9.2 & 3 \\
\hline K86 & Hypertension uncomplicated & 1124 & 6.2 & 4 \\
\hline $\mathbf{R} 80$ & Influenza & 638 & 3.5 & 5 \\
\hline T90 & diabetes mellitus & 589 & 3.3 & 6 \\
\hline D70 & Gastrointestinal infection & 324 & 1.8 & 7 \\
\hline U71 & Cystitis/Urinary infection other & 285 & 1.6 & 8 \\
\hline F70 & Conjunctivitis infectious & 276 & 1.5 & 9 \\
\hline L90 & Osteoarthritis of knee & 272 & 1.5 & 10 \\
\hline S76 & Skin infection other & 252 & 1.4 & 11 \\
\hline B82 & Anaemia other/unspecified & 232 & 1.3 & 12 \\
\hline L20 & J oint symptom/complaint NOS & 221 & 1.2 & 13 \\
\hline H70 & Otitis externa & 220 & 1.2 & 14 \\
\hline A97 & No disease & 218 & 1.2 & 15 \\
\hline S11 & Skin infection post traumatic & 208 & 1.1 & 16 \\
\hline D22 & Worms / Parasites & 195 & 1.1 & 17 \\
\hline D08 & Flatulence/gas/belching & 193 & 1.1 & 18 \\
\hline L86 & Back syndrome with radiating pain & 188 & 1.0 & 19 \\
\hline L91 & Osteoarthritis others & 163 & 0.9 & 20 \\
\hline \multicolumn{2}{|c|}{ All other diagnoses } & 5965 & 33.0 & \\
\hline \multicolumn{2}{|l|}{ Total } & 18075 & 100.0 & \\
\hline
\end{tabular}

\section{The Top five health managed problems by I CPC chapters}

We have taken into consideration the top eight chapters that included about $90 \%$ of all health problems which are managed by the GPs. Additional material, shows the most frequent managed problems (Top 5) in each of these chapters.

\section{Distribution of health problems by sex}

Respiratory diseases came in first place for both genders. However, skin diseases, which are ranked second, were more prevalent in males and digestive problems in females. The genital affections and psychological problems were at the bottom of the list, making them the less identified affections in primary health care.

\section{Distribution of health problems by age group}

The distribution of problems managed (additional material) was variable with age. Up to 24 years, the top three identified problems were respiratory affections, such as tonsillitis acute, upper respiratory infection, and acute bronchitis. Among children, under 5 years of age, the frequency of respiratory diseases was more than $60 \%$, whereas digestive affections, such as diarrhoea, gastrointestinal infections, worms and parasites, came next and constituted 14\%. For adults 25-44 years of age, respiratory affections remained the most frequent problem (34\%), but high blood pressure was among the top $10(1.6 \%)$ in ninth position. 
For the age group of 45-64 years, uncomplicated hypertension was the top health problem (14.2\%), followed by diabetes (9.3\%) and acute bronchitis $(8.5 \%)$. In this age group, locomotive (musculoskeletal) affections became frequent and constituted the fourth group of managed morbidity $(10.2 \%)$.

Elderly population aged 65 years and over was characterized by an increase of chronic affections. High blood pressure was the most common disease $(22.4 \%)$ and diabetes mellitus came in third place $(7.9 \%)$. In addition, respiratory affections and locomotive illnesses were also frequent and presented, together, almost $30 \%$.

\section{Discussion}

\section{Strengths and limitations of the study}

This is the first time such a wide morbidity study had been conducted in Tunisia. The results enabled us to identify the main health problems managed in primary health care in the province of Sousse. However, these results were mainly representative of morbidity at the public health sector because no private health settings have been included in this study. We also did not take into account the prevention activities, such as immunisation, ante-natal care and family planning, which are mainly being managed by nurses and midwives in these health centres.

In addition, some health problems could not be classified and were thus coded as "other." This was mainly noted in skin, digestive, and locomotive affections.

\section{Characteristics of general practice attendees}

Sex distribution showed that $62 \%$ of patients were females. The predominance of the female population was reported in other developed and developing countries [2-5]. Female predominance, which is universal, is significant and could be explained by the fact that women are more anxious of their health, or maybe more vulnerable because of their reproductive health life (e.g., child bearing, genital activities, post partum). In addition, they are probably more available than men to attend the health centres or more able to manage the long waiting time at the health settings $[2,3]$.

Age distribution showed that one third of people attending general primary care consultations were children under 15 years and that half was aged under 25 years, which is relatively similar to the age distribution in the general population $(49.2 \%)$. The elderly population aged 60 years and over constituted $17.6 \%$ of the total, which is significantly higher than in the general population $(8.9 \%, \mathrm{P}<0.05)$. Similar findings were reported in other countries [2]. The predominance of male children, especially, under five was noted in many other studies, particularly in countries that have similar cultures to that of Tunisia, where boys are still more welcome than girls $[6,7]$.

\section{Medical consultation}

It was found that the great majority of consultations were for the first episode of a health problem (81\%) rather than for control or monitoring of existing conditions $(5.5 \%)$, which indicated that the demand for care, in general practice, was mainly for acute illnesses as reported in other studies $[5,6]$.

The average consultation time was 6.8 minutes $( \pm 3.8)$ (mode $=5$ minutes), and the duration varied from 1 to 30 minutes. Many other studies were interested in the length of consultation. In Estonia [9] for example, the average consultation lasted 9 minutes ( \pm 4.9 ) and varied according to the number and the nature of problems, as well as with patient's age, but was not influenced by gender. According to the same study, physical examination lasted about 2 minutes $( \pm 1.9$ ) and consultation time was longer for older age groups and for patients with psychological problems. In France, most of consultations in general practice lasted between 10 and 20 minutes, and 92\% lasted less than 25 minutes (15 minutes: $37 \%$, 10 minutes: $23 \%, 20$ minutes: 19\%). It has been also shown that the duration increased for chronic diseases, as well as for elderly people, and with female doctors [9]. 


\section{Patterns of problems managed in general practice}

\section{The most common problems managed}

Health problems in the region of Sousse classified as "diagnoses" according to ICPC chapters showed that respiratory diseases were the most prevalent problem (43\%). This finding is universally common as similar findings have been reported in many other studies [2, 4-5, 10-12], except in China, where high blood pressure and atherosclerosis ranked first [4]. The high proportion of respiratory diseases that have been noted in our study should be taken seriously in order to find out the main reasons of this predominance.

Some questions should be asked, such as, how are respiratory affections being taken care of in general practice, and how do GPs manage the use of antibiotics in the treatment of respiratory diseases? Theses questions are important because GPs tend to prescribe antibiotics for respiratory affections based on clinical arguments or merely to satisfy patients' demand for antibiotic prescriptions [13]. As reported by Belgith [26], antibiotics were prescribed by GPs in the region of Monastir (Tunisia) to $50.4 \%$ of consulting patients of whom $52.8 \%$ did not require laboratory tests. The number of prescribed medicines as well as the non-respect of the therapeutic instructions constituted a potentially dangerous practice. This should justify the development of specific guidelines and training curricula for GPs, as well as vocational training courses that should be regularly assessed and updated [11]. Because tobacco use is high among young people and adults in Tunisia (20-40\%), GPs should focus on educating people about the harmful effects of tobacco addiction and passive smoking on health.

A study conducted, in Souses [14], on physicians' attitude towards tobacco use and prevention, concluded that their interest in health education regarding the harmful effects of tobacco depended on whether tobacco was involved in the current patient's episode of care, and only $17.2 \%$ of physicians spontaneously provided advice to their patients on smoking cessation.

\section{The most uncommon problems managed}

Male and female genital affections accounted for only $1 \%$ of visits. Similar results were found in many other studies [2, 4, 12]. This low percentage could be explained by the presence of a midwife in each health centre, indicating that women may prefer them to male GPs. This issue also could be explained by the fact that genital complaints, in our culture, were often shyly declared and the GPs did not make the necessary effort to look beyond the declared symptom in order to unveil the hidden main reason for the encounter [15]. It was reported in other studies that this issue was related to a lack of doctor-patient communication or to an overcharge of medical consultations, hindering the quality of patient's monitoring in primary health centres $[3,17]$.

It was also noted that social and psychological problems did not frequently emerge either as reason for encounter or as identified problem by the GPs $(0.85 \%)$. This finding has also been noted in many other studies [2, 4, 12, 15]. Nandani de Silva [2] found that psychological problems accounted for only $1.9 \%$ in Sri Lanka, which was similar to findings in a Hong Kong survey [16]. According to an Australian survey, psychological illnesses accounted for $5.9 \%$ of diagnosed problems [16]. Surveys on psychiatric morbidity worldwide have shown that psychological problems, which accounted for about a third of all general practice consultation [2], were often missed by GPs. It was shown that GPs' failure to detect psychological illnesses resulted because patients, more often, somatise their illness and express non specific complaints, which would be classified under other organic ICPC chapters and rubrics [2]. We should also note that the GPs were lacking specific training on mental health issues, so they rely on specialists to take care of patients presenting such illnesses. This argument would be confirmed as we know that Tunisian physicians have been trained according to a biomedical model, which had largely disregarded the psychological and social aspects of the health problem. It has been clearly shown that this issue constitutes a deep lack in general practice and that GPs need to enhance their knowledge and skills in this field $[17,18]$.

\section{Health problems by age group}

Health problems managed in general practice were variable with age $[2,19]$. Children under five years of age, as shown in many other studies, were mainly suffering from respiratory diseases, such as upper respiratory tract infections, acute bronchitis, and tonsillitis acute [6, 7]. However, 
we should note that cardiovascular diseases (CVD), such as high blood pressure and diabetes mellitus started to appear early among young adults. In the age group of $25-44$, these two chronic diseases were present, at the rates of $1.6 \%$ and $2.1 \%$, respectively. In the age group of $45-64$ years, uncomplicated hypertension came first (14.2\%), followed by diabetes (9.3\%). For elderly people aged 65 years and over, the occurrence of CVD became almost similar to the one in developed countries $[4,8,19,20]$. This finding showed that the Tunisian population has been experiencing an epidemiological transition, where CVD are starting early and increasing in the general population. According to a recent study which aimed to assess CVD risk factors in urban schoolchildren in Sousse (Tunisia) [21], school children were exposed early to CVD risk factors, such as obesity $(7.9 \%)$ and smoking $(7.6 \%)$. It was also noted that among young people, high blood pressure and lipid disorders increased significantly with the weight. Therefore, the health system should be aware of the current and long term consequences of these diseases, such as degenerative complications and the increase of cardiovascular morbidity and mortality. Health managers should plan to set up preventive programmes and procedures for early diagnosis of cardiovascular affections [22], and GPs should be able to ensure health promotional activities, particularly for young people, to make them aware of the danger of cardiovascular risk factors related to people' attitudes and behaviours, such as fast-food consumption, lack of physical exercise, and tobacco use $[22,23]$. Therefore, reducing the prevalence and the incidence of cardiovascular morbidity and mortality should be done through an effective involvement of GPs in relevant community projects especially designed for preventive and promotional care [24].

\section{Conclusion}

Primary health has very specific patterns that are different from what medical students are currently facing in hospitals during their vocational training. GPs in primary health care deal with a large scope of illnesses, including chronic diseases as well as preventive and promotional care. Therefore, we should engage a serious reflection on what the necessary scientific core curriculum should be for GPs and how this can help to improve the skills of our future GPs. [25, 27-29]. The findings will also interest health policy makers in Tunisia and regional primary health programme makers.

\section{Competing interests}

This survey was achieved with the support of the Research Unit «PMA 01/UR/08-15 》 - Department of community medicine - Faculty of Medicine of Sousse.

\section{Authors' contributions}

AM (Chief of Department) developed the research protocol, made contacts with local authorities and laid the groundwork for the realisation of the field work. He also trained the team of the department for data coding and statistical analysis of results. RG, TA, and IB conducted in-depth bibliography review on morbidity in primary health care and participated in the monitoring of data collection, training of health workers in primary health care centres included in this study (doctors, nurses and centre managers). They also participated in the process of data coding, computer data entry and data quality control. They performed data analysis and interpretation of results and contributed to the write up of the manuscript. RG supervised the article writing process, and all authors have read and approved this manuscript before submission and subsequent revisions.

\section{Acknowledgement}

This survey was achieved with the support of the Research Unit «PMA 01/UR/08-15 » Department of community Medicine - Faculty of Medicine of Sousse. 


\section{References}

1. Jamoulle M, Roland M. Classification Internationale des Soins Primaires. Editions Alexandre Lacassagne, Lyon, 1992

2. Silva N, Mendis K. One day general practice morbidity survey in Sri Lanka. Family Practice 1998; 15: 323-331

3. Soltani MS, Letaief M, Ben Salem K, Mandhouj O, Gacem H, Belghith Z, Nouira R, Bchir A. Motifs de contacts au niveau de première ligne dans le Sahel tunisien. Arch. Public Health 2002, $60: 125-140$

4. Wun YT, Lu XQ, Liang WN. The work by the developing primary care team in China: a survey in two cities. Family Practice 2000; 17:10-15

5. Wun YT, Wong TW, Tam W, Yu TS. Patient characteristics of encounters in general practice. The Hong Kong Pract. 2002; 24: 59-65

6. Mennerat $\mathrm{F}$, Lamberts $\mathrm{H}$, Okkes I. Dossier de patient structure en épisodes et analyse des données en médecine générale 2 . Les perspectives en France. Informatique et Santé $1999 ; 11: 225-230$

7. Tursz $A$, Crost $M$, Kermani $S$. Etude épidémiologique du recours aux soins curatifs des enfants de moins de 5 ans en Algérie : quelles leçons pour le système de santé ? Rev. Epidém. et Santé Publ. $1999 ; 47$ : 2S18-2S37

8. Andrieu S, Grand A. Epidémiologie du vieillissement, un gain d'autonomie ? Concours Médical 2000 ; Tome 122-38 : 5-8

9. Tahepold H, Maaroos HI, Kalda R, Van den Brink-Muinen A. Structure and duration of consultations in Estonia. Scand J Print Health Care 2003; 21

10. Campion PD, Gabriel J. Child consultation patterns in general practice comparing "high" and "low" consulting family. BMJ 1984; 288: 1426-1428

11. Dickinson JA, Chan CSY. Antibiotic use by practitioner in Hong Kong. The Hong Kong practitioner 2002 June ; vol. $24: 15$ p.

12. Pearson N, Jim OB, Huw T. Collecting morbidity data in general practice: the Somerest morbidity project. BMJ 1996; 312: 1517-1520

13. Britten N. Patients' demands for prescriptions in primary care. BMJ 1995; $310: 1084-1085$

14. Mtiraoui A, Bougmiza I, Braham M, Gataa R, Bouraoui O, Letaief M, Derbali M, Haj Fredj A, Ajmi Th. Attitudes des médecins de la région de Sousse vis-à-vis de la lutte anti-tabac. Microb. Hyg. Alim. Vol 15, N 44, Décembre 2003

15. Goriaux J L. Pour une pratique psychothérapeutique en médecine générale. La revue du praticien - médecine générale $1998 ; 12$ (428) : 28-30

16. Cheung KY. "Tell me about your most loyal patients". Doctors perceptions on factors affecting the doctor-patients relationship. The Hong Kong practitioner 2003 January; volume $25: 15 \mathrm{p}$

17. Bleuez S. Ecouter derrière le symptôme. La revue du praticien-médecine générale $2000 ; 14$ (483) : 48-49

18. O'toole B, Driver B, Britt H, Bridges WC. Using general practitioners to measure community morbidity. International journal of epidemiology 1991; 20 (4) : 11251132

19. Chabrun-Robert C. Etat de santé et recours aux soins en 1995. Concours Médical $1997 ; 119-07$ : 480-482

20. Chaubrun-Robert C. Vieillissement de la population et dépenses de santé. Concours Médical $1998 ; 120-33: 2335-2336$

21. Ghannem H, Darioli R, Limam K, Harrabi I, Gaha R, Trabelsi L, Haj Fredj A, Bouslama A. Epidemiology of cardiovascular risk factors among schoolchildren in Sousse, Tunisia. J ournal of Cardiovascular Risk 2001; 8: 87-91

22. Charlton BG, Calvert N, White M, Rye GP, Conrad W, Van Zwanenberg T. Health promotion priorities for general practice: constructing and using « indicative prevalence ». BMJ 1994; $308:$ 1019-1021

23. Beraud C. Pourquoi et comment accroître la responsabilité des médecins généralistes. Concours Médical 1994; 116-01 : 58-61

24. Sarradon-Eck A. La rencontre médecin-malade est aussi le lieu d'une médiation du lien social. La revue du praticien - médecine générale 2002 ; 16 ( 578 ): $938-943$

25. Draperi C. L'indispensable place des sciences humaines dans l'exercice de la médecine générale. Revue du Praticien- Médecine générale $2002 ; 16$ (587) : 1438 1439

26. Belgith Z et al. The antibiotherapy in general medicine in Monastir, Tunisia. Eastern Mediterranean Health Journal; Volume 5, Issue 2, 1999, P $328-332$.

27. Menken M. Préparer les futurs médecins aux soins de santé primaires. Forum mondial de la santé $1991 ; 12: 396-398$

28. Olesen F, Dikinson J, Hjortdahl. General practice - time for a new definition. BMJ Volume: 320, 5 February 2000

29. Ruth MH, Grey P, Asthon JR. Training doctors for the year 2000. Medical Education 1990; 24, 11-13 